\title{
Bronchial thermoplasty guided by hyperpolarised gas magnetic resonance imaging in adults with severe asthma: a 1-year pilot randomised trial
}

\author{
Sarah Svenningsen ${ }^{1,2}$, Parameswaran Nair ${ }^{1,2}$, Rachel L. Eddy ${ }^{3,4}$, Marrissa J. Mclntosh ${ }^{3,4}$, \\ Melanie Kjarsgaard ${ }^{1}$, Hui Fang Lim ${ }^{1}$, David G. McCormack ${ }^{5}$, Gerard Cox $\mathbb{D}^{1,2}$ and Grace Parraga ${ }^{3,4}$
}

${ }^{1}$ Firestone Institute for Respiratory Health, St Joseph's Healthcare, Hamilton, Canada. ${ }^{2}$ Dept of Medicine, Division of Respirology, McMaster University, Hamilton, Canada. ${ }^{3}$ Robarts Research Institute, Western University, London, Canada. ${ }^{4}$ Dept of Medical Biophysics, Western University, London, Canada. ${ }^{5}$ Dept of Medicine, Division of Respirology, Western University, London, Canada.

Corresponding author: Sarah Svenningsen (svennins@mcmaster.ca)

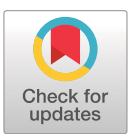

Copyright $\odot$ The authors 2021

This version is distributed under the terms of the Creative Commons Attribution NonCommercial Licence 4.0. For commercial reproduction rights and permissions contact permissions@ersnet.org

This article has supplementary material available from openres.ersjournals.com

Received: 18 April 2021 Accepted: 2 July 2021

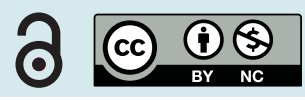

Shareable abstract (@ERSpublications)

In adults with severe asthma, MRI-guided BT reduces the number of radiofrequency activations and bronchoscopy sessions, and results in asthma quality-of-life and control improvements at 12 months that are non-inferior to standard whole-lung BT https://bit.ly/3qWYjp4

Cite this article as: Svenningsen S, Nair P, Eddy RL, et al. Bronchial thermoplasty guided by hyperpolarised gas magnetic resonance imaging in adults with severe asthma: a 1-year pilot randomised trial. ERJ Open Res 2021; 7: 00268-2021 [DOI: 10.1183/23120541.00268-2021].

\section{Abstract}

Patient-specific localisation of ventilation defects using hyperpolarised gas magnetic resonance imaging (MRI) introduces the possibility of regionally targeted bronchial thermoplasty (BT) for the treatment of severe asthma. We aimed to demonstrate that BT guided by MRI to ventilation defects reduces the number of radiofrequency activations while resulting in improved asthma quality-of-life and control scores that are non-inferior to standard BT.

In a 1-year pilot randomised controlled trial, 14 patients with severe asthma who were clinically eligible to receive BT underwent hyperpolarised gas MRI to characterise ventilation defects and were randomised to MRI-guided or standard BT. End-points were improved Asthma Quality of Life Questionnaire (AQLQ) and Asthma Control Questionnaire (ACQ) scores, the proportion of AQLQ and ACQ responders and the number of radiofrequency activations and bronchoscopy sessions.

Participants who underwent MRI-guided BT received 53\% fewer radiofrequency activations than those who had standard BT ( $\mathrm{p}=0.003$ ). At 12 months, the mean improvement from baseline was similar between the MRI-guided group $(n=5)$ and the standard group $(n=7)$ for AQLQ score (MRI-guided: 1.8, 95\% CI 0.1-3.5, $\mathrm{p}=0.04$; standard: 0.7, 95\% CI -0.9-2.3, $\mathrm{p}=0.30)(\mathrm{p}=0.25)$ and ACQ-5 score (MRI-guided: -1.4 , 95\% CI $-2.6--0.2, \mathrm{p}=0.03$; standard: $-0.7,95 \% \mathrm{CI}-1.3-0.0, \mathrm{p}=0.04)(\mathrm{p}=0.17)$. A similar proportion of participants in both groups achieved a clinically relevant improvement in AQLQ score (MRI-guided: 80\%; standard: 71\%) and ACQ-5 score (MRI-guided: 80\%; standard: 57\%).

Hyperpolarised gas MRI-guided BT reduced the number of radiofrequency activations, and resulted in asthma quality of life and control improvements at 12 months that were non-inferior to standard BT.

\section{Introduction}

Variable airway narrowing and airflow obstruction due to airway smooth muscle (ASM) dysfunction are diagnostic features of asthma that contribute to symptoms and disease severity. Bronchial thermoplasty (BT) has been developed as a non-pharmacological procedure that targets the ASM component of asthma [1]. Histological studies confirm that local intraluminal delivery of radiofrequency energy to the walls of airways that are accessible by a bronchoscope results in reduced ASM mass [2-5]. BT of all accessible airways, performed over three bronchoscopy sessions, elicits a prolonged clinical benefit that has been confirmed in clinical trials [6-11] and real-world studies [11, 12]. Despite safety and efficacy profiles [6-11] sustained for 10 years or more [13], most guidelines do not fully endorse BT and its clinical uptake has been slow, mainly because it remains difficult to predict which patients will respond to BT and the 
procedure requires endoscopy time and costs $[14,15]$ and involves risk of worsening asthma and other adverse events. Strategies that reduce the number of BT activations and bronchoscopy sessions may help to curb some of these limitations.

In asthma, airway abnormalities are patient specific, heterogeneously distributed and tend to persist and reoccur in the same location over time [16]. Hence, it is possible that conventional BT may be treating airways that do not contribute to patient symptoms or disease severity. Patient-specific localisation of focal airway abnormalities introduces the possibility of regionally targeted BT, which has the advantage of avoiding treatment of normal airways.

Focal ventilation defects that can be visualised using hyperpolarised gas magnetic resonance imaging (MRI) have been shown to be the functional consequence of luminal narrowing [17] and obstruction by mucus and eosinophils [18, 19]. Related to the pathophysiology of ASM dysfunction, MRI ventilation defects respond in the anticipated direction following bronchoconstriction [20-22] and bronchodilation [20, 23]. They also correlate with disease severity [24] and are predictive of asthma exacerbations [25] and control [26].

We and others [19, 27] postulated that BT could be optimised by treating only those focal airway abnormalities that exhibit abnormal MRI ventilation. Pilot results of MRI-guided BT showed that after 3 months there was no difference in Asthma Quality of Life Questionnaire (AQLQ) score improvement after a single session of MRI-guided BT as compared to the conventional three-session approach [28]. We hypothesised that MRI-guided BT would result in a reduced number of radiofrequency activations and bronchoscopy sessions. We also postulated that 1 year after the end of therapy, AQLQ and five-item Asthma Control Questionnaire (ACQ-5) score improvements would be similar between MRI-guided and standard whole-lung BT. Here we report the results of a 12-month pilot randomised controlled trial comparing MRI-guided and standard BT in severe asthma.

\section{Material and methods}

\section{Study design and participants}

We conducted a randomised controlled trial of hyperpolarised gas MRI-guided versus standard BT (1:1 allocation ratio) at two sites (London Health Sciences Centre, Western University, and St. Joseph's Healthcare Hamilton, McMaster University, Canada) between October 2014 and November 2019 (ClinicalTrials.gov NCT02263794). Study participants were $>18$ years of age with severe asthma that was poorly controlled [30], and BT was prescribed as part of their clinical care. Written informed consent to ethics board-approved protocols (University of Western Ontario Health Sciences Research Ethics Board (\#104200), Hamilton Integrated Research Ethics Board (\#14-642)) was obtained from all participants.

As shown in figure 1, the trial comprised a pre-intervention period, an intervention period and a post-intervention follow-up period of 12 months. During the pre-intervention period, all participants completed a study visit before (baseline, visit 1) and after receiving an oral corticosteroid (OCS) burst (post-OCS burst, visit 2). During the post-intervention period, all participants were managed according to standard of care; follow-up visits were completed by telephone or in person at 3 (visit 3), 6 (visit 4) and 12 months (visit 5) after MRI-guided or standard BT was completed. At face-to-face visits, spirometry and hyperpolarised gas MRI were performed pre- and post-bronchodilator. The AQLQ and ACQ-5 were completed for all telephone and face-to-face visits. For post-bronchodilator measurements, four $100 \mu \mathrm{g}$ doses of salbutamol (Teva-Salbutamol HFA, Teva Canada Ltd.) were delivered through a pressurised metered dose inhaler using an AeroChamber Plus spacer (Trudell Medical International).

\section{Image acquisition and analysis}

All imaging was completed at the Robarts Research Institute (Western University, London, Canada). ${ }^{1} \mathrm{H}$ and inhaled hyperpolarised gas $\left({ }^{3} \mathrm{He}\right.$ and/or $\left.{ }^{129} \mathrm{Xe}\right)$ MR images $\left(3 \times 3 \times 15 \mathrm{~mm}^{3}\right)$ were acquired within $5 \mathrm{~min}$ of one another using a 3T MR system (General Electric Health Care, Milwaukee, WI, USA) as previously described [23]. For all acquisitions, participants were coached to inhale $1 \mathrm{~L}$ of gas from functional residual capacity (FRC), and coronal slices were acquired under breath-hold conditions at FRC+1 L. Thoracic computed tomography (CT) was performed at a similar lung volume using a 64-slice Lightspeed VCT system (GE Healthcare) as previously described [23]. Quantitative MRI analysis was performed by a single trained observer who was blinded to intervention allocation (MM) using semi-automated segmentation and registration software to generate the MRI ventilation defect per cent (VDP) at baseline [31]. The observer segmented each baseline MRI dataset twice (coefficient of variation 14.9\%; intraclass correlation coefficient 0.97 , 95\% CI 0.93-0.99), and the mean VDP of the two rounds was reported. 


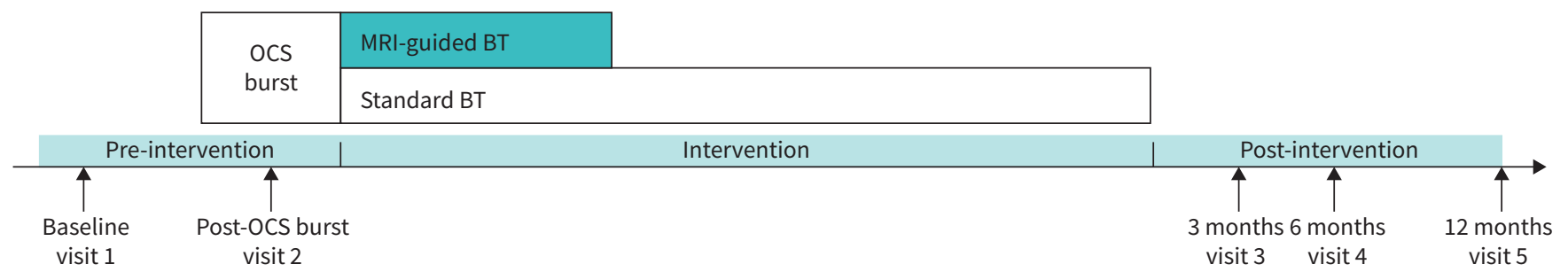

FIGURE 1 Study design. All participants completed two pre-intervention study visits before being randomised to magnetic resonance imaging (MRI)-guided or standard bronchial thermoplasty (BT). For those participants randomised to MRI-guided BT, target airways were identified on inhaled hyperpolarised gas MRI acquired at baseline (visit 1) and following an oral corticosteroid (OCS) burst (visit 2). The post-intervention follow-up period was 12 months, with outcome assessment visits completed 3 (visit 3), 6 (visit 4) and 12 months (visit 5) after BT.

\section{Bronchial thermoplasty}

BT was performed using the Alair Bronchial Thermoplasty System (Boston Scientific, Marlborough, MA, USA) by bronchoscopists (DGM and GC) with >10 years' experience [6]. All participants received a 5-day OCS burst (50 mg of prednisone/day) prior to BT. Participants randomised to standard BT underwent three bronchoscopy procedures to treat all accessible airways as previously described [1,32]. Those who were randomised to MRI-guided BT underwent up to two bronchoscopy procedures to treat all accessible airway targets visually identified by the bronchoscopy team on hyperpolarised gas MRI acquired at pre-intervention visits. As shown in figure 2, bronchopulmonary segments were considered targets if they demonstrated properties of smooth muscle dysfunction, characterised by their spatial relationship to ventilation defects that were completely or partially reversible following bronchodilator inhalation [19] at one or both pre-intervention visits. The bronchopulmonary segment that spatially corresponded to a reversible ventilation defect was identified by the bronchoscopists based on their knowledge of the bronchopulmonary segmental anatomy. CT scans were available for review alongside the MRI as a structural aid, but they were not co-registered to the MRI or segmented to delineate the bronchopulmonary segments.

\section{Outcomes}

Our primary outcome was the change in AQLQ score between baseline (visit 1) and follow-up visits completed 3 (visit 3), 6 (visit 4) and 12 months (visit 5) after BT. Secondary outcomes were change in ACQ-5 score, the percentage of participants achieving AQLQ and ACQ-5 score improvements $\geqslant$ minimal clinically important difference (MCID $\geqslant 0.5[33,34]$ ) and the difference in the number of radiofrequency activations and bronchoscopy sessions between intervention groups. All participants were monitored for adverse events related to hyperpolarised gas MRI, and clinically for adverse respiratory events during the intervention period plus 6 weeks and post-intervention period (6 weeks to 12 months).

\section{Statistical analysis}

Because this was a pilot trial, we did not complete an a priori sample size calculation; based on our previous observation of differences in AQLQ and ACQ-5 improvements between two groups of BT patients [35], we recruited 14 patients to this study. Data were tested for normality using the Shapiro-Wilk normality test and when data were not normal, nonparametric tests were performed. Baseline and follow-up AQLQ and ACQ-5 scores were compared using paired t-tests and Wilcoxon matched-pairs signed rank tests. The difference between groups for baseline demographics, clinical characteristics, the number of radiofrequency activations and the change in AQLQ and ACQ-5 scores between baseline and follow-up were evaluated using unpaired t-tests and Mann-Whitney U-tests. We also determined the proportion of participants in each group achieving AQLQ and ACQ-5 score improvements ₹MCID [33, 34]. Fisher's exact tests were performed to determine if these proportions were statistically different. Data are expressed as mean \pm SD or mean (95\% CI). Intention-to-treat (supplementary material) and per-protocol analyses were performed. All statistical analyses were performed using GraphPad Prism v7.00 (GraphPad Software Inc., San Diego, CA, USA) or SPSS v23.00 (IBM Corporation, Armonk, NY, USA).

\section{Results}

In total, 14 participants completed the pre-intervention period and were randomised to MRI-guided BT $(n=6)$ or standard BT $(n=8)$ (figure 3). After randomisation, two participants were excluded following one or more BT sessions; one participant was initiated on anti-interleukin-5 monoclonal antibody therapy and the other was unable to complete BT owing to the coronavirus disease 2019 pandemic. In total, five 


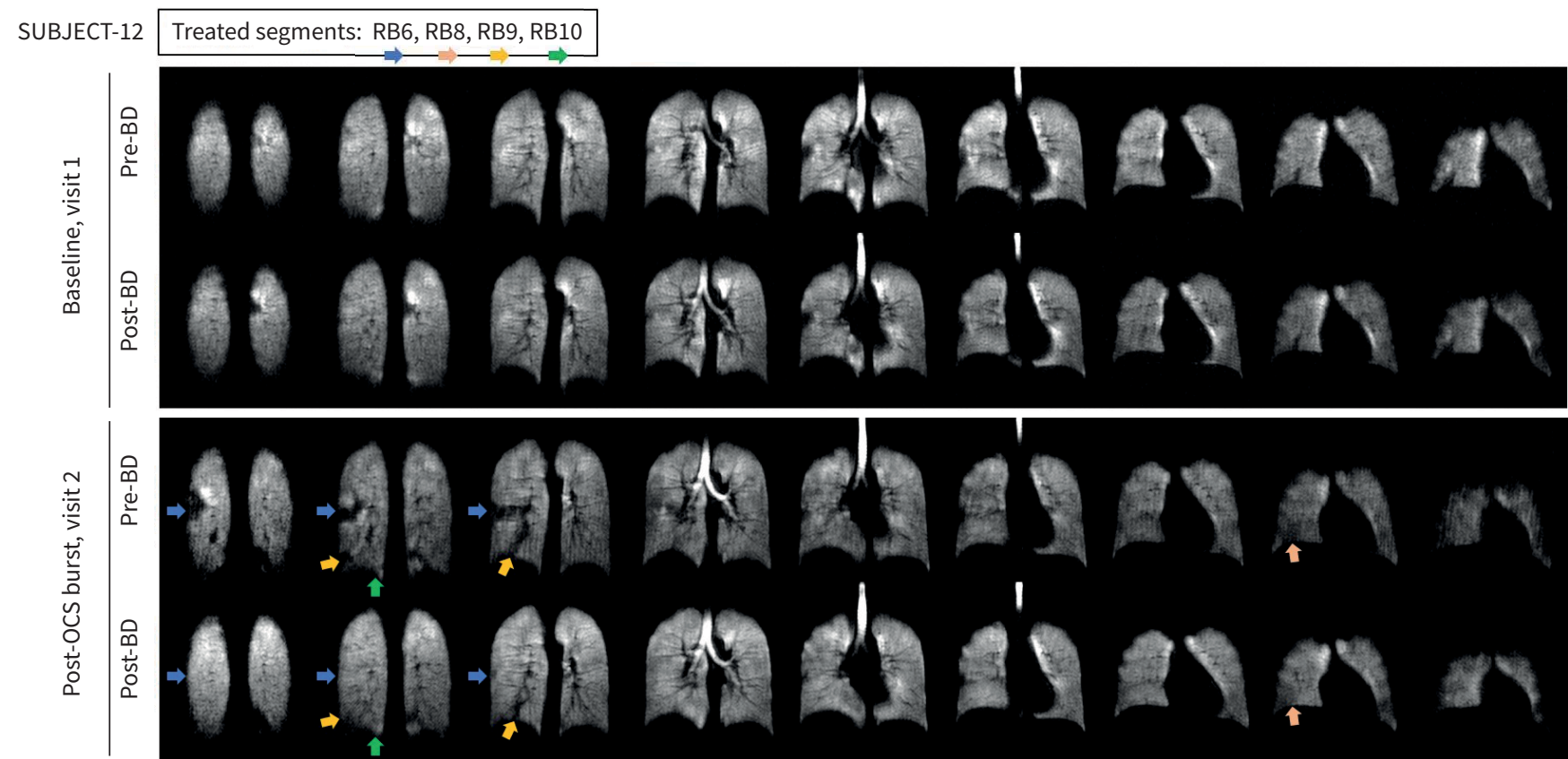

SUBJECT-23 Treated segments: RB1, RB8, RB10, LB7+8, LB10

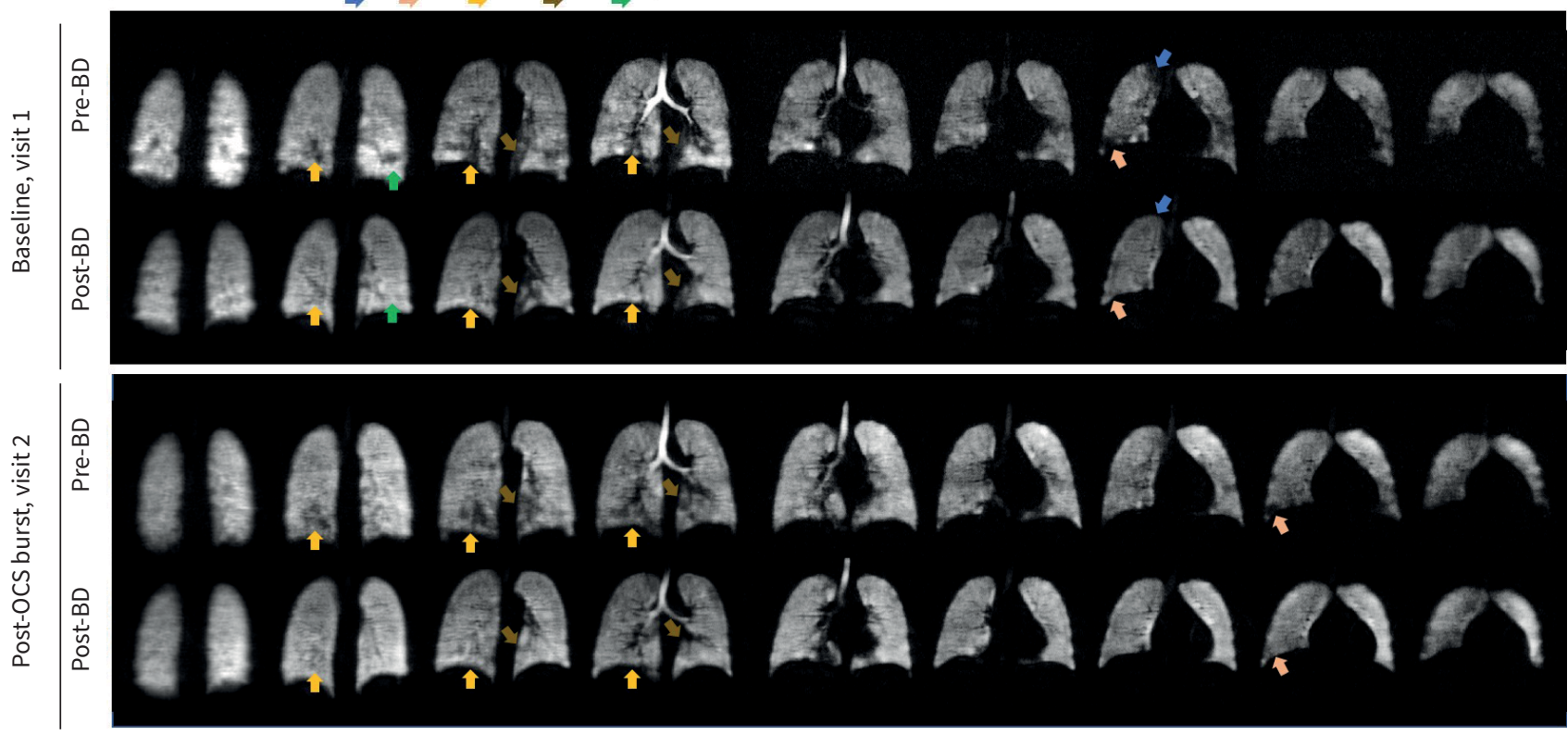

FIGURE 2 Representative examples of focal airway targets identified by inhaled hyperpolarised gas magnetic resonance imaging (MRI). Pre- and post-bronchodilator (BD) inhaled hyperpolarised gas MRI coronal slices acquired during the pre-intervention period at baseline (visit 1 ) and after receiving an oral corticosteroid (OCS) burst (visit 2) for two representative participants with severe asthma. Arrows identify focal ventilation defects that were deemed targets by the bronchoscopist because they were completely or partially reversible following bronchodilator inhalation at one or both pre-intervention visits. For subject-12, the RB6, RB8, RB9 and RB10 bronchopulmonary segments were treated during a single MRI-guided bronchial thermoplasty (BT) session. For subject-23, the RB1, RB8, RB10, LB7+8 and LB10 bronchopulmonary segments were treated during a single MRI-guided BT session.

participants who underwent MRI-guided BT and seven participants who underwent standard BT were included in the per-protocol analysis.

Baseline demographics and characteristics

Baseline demographics and clinical characteristics for participants in the per-protocol analysis are summarised in table 1. The intervention groups were similar except for forced expiratory volume in $1 \mathrm{~s}\left(\mathrm{FEV}_{1}\right)$, 


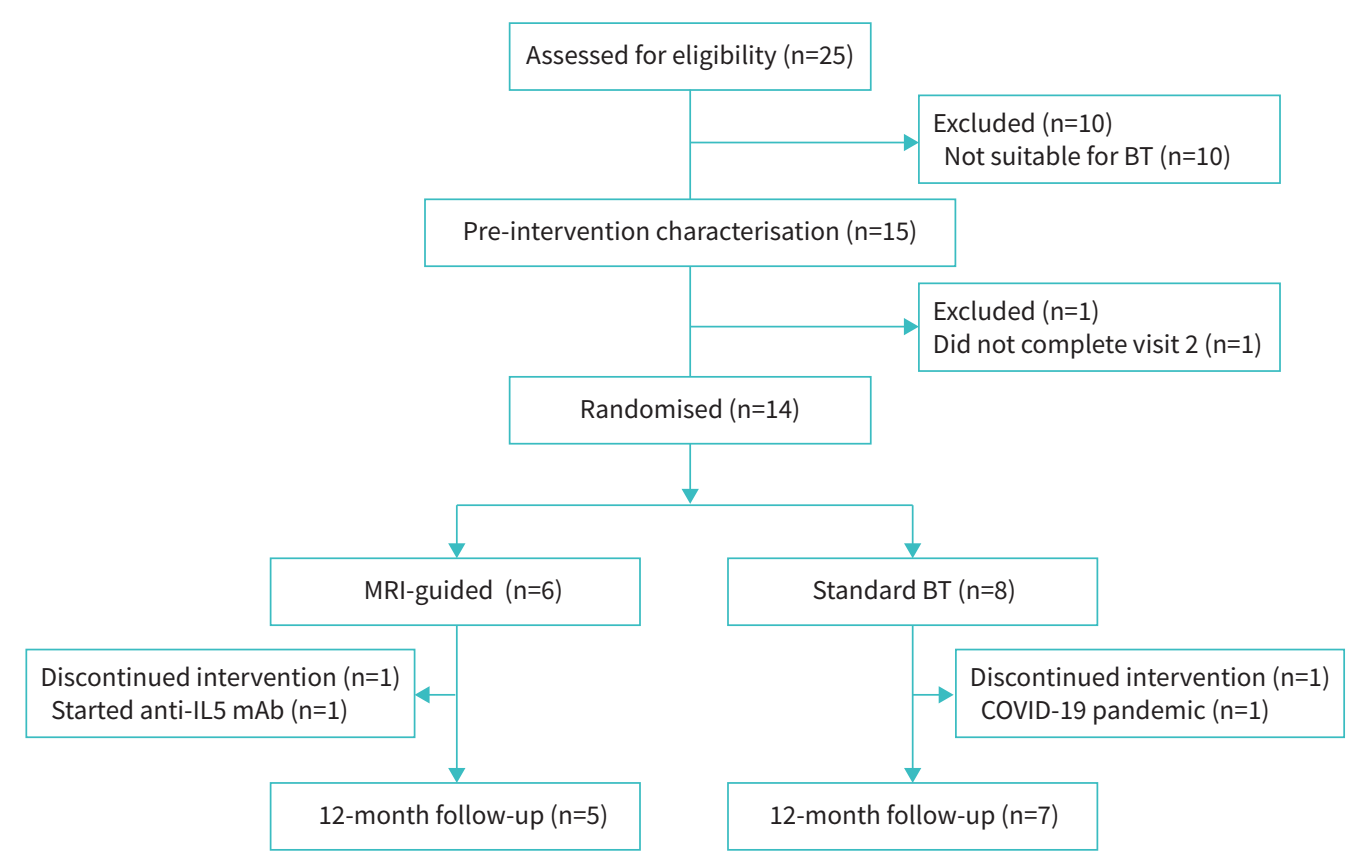

FIGURE 3 Consolidated Standards of Reporting Trials (CONSORT) diagram. BT: bronchial thermoplasty; MRI: magnetic resonance imaging; IL-5 mAb: interleukin 5 monoclonal antibody; COVID-19: coronavirus disease 2019.

which was lower in the MRI-guided $\mathrm{BT}$ group, and $\mathrm{FEV}_{1}$ reversibility, which was greater in the MRI-guided BT group. One participant (20\%) in the MRI-guided BT group was OCS dependent, whereas two participants (29\%) in the standard BT group were OCS dependent. Three participants (60\%) in the MRI-guided BT group were receiving biologic therapy (omalizumab, $\mathrm{n}=1$; mepolizumab, $\mathrm{n}=1$; reslizumab, $\mathrm{n}=1$ ) as compared to one participant $(14 \%)$ in the standard BT group (mepolizumab, $\mathrm{n}=1$ ). Airway inflammation (characterised using sputum cell counts) was controlled prior to BT for four participants (80\%) in the MRI-guided BT group and five participants $(71 \%)$ in the standard BT group. In the remaining three participants, airway inflammation was not characterised prior to BT; however, their blood eosinophil counts were within the normal range. The burden of mucus plugging was low in both groups, with intraluminal plugging visualised on CT in three bronchopulmonary segments for one participant in each group.

\section{Activation outcomes}

Participants who underwent MRI-guided BT received significantly fewer $(73 \pm 37, p=0.003$ ) radiofrequency activations than those who underwent standard BT (155 \pm 34$)$. The median number of bronchopulmonary segments treated was seven (minimum of four, maximum of ten) in the MRI-guided BT group and 18 (minimum of 13, maximum of 18) in the standard BT group. For participants in the MRI-guided BT group, all target airways were treated in a single bronchoscopy session for three participants (four, six and seven segments treated), while two participants required two bronchoscopy sessions to treat all targets (nine and ten segments treated) (seven sessions in five MRI-guided BT participants (47\% of standard BT)). Participants requiring two bronchoscopy sessions had more target airways identified by the bronchoscopist, who also determined that treatment of all airway targets required two BT sessions. All participants in the standard BT group underwent three bronchoscopy procedures to treat all accessible bronchopulmonary segments (21 sessions in seven standard BT participants (100\% of standard BT)).

\section{Asthma quality of life and control outcomes}

Figure 4 summarises asthma quality of life and control outcomes at 3, 6 and 12 months by intervention group. In supplementary figure S1, inter-individual changes in AQLQ and ACQ-5 scores are shown from baseline to 12 months post-BT. As shown in figure 4a and supplementary figure S1A, the mean change in AQLQ score from baseline to 12 months post-BT was 1.8 (95\% CI $0.1-3.5)(n=5, p=0.04)$ in the MRI-guided BT group and 0.7 (95\% CI -0.9-2.3) $(n=7, p=0.30)$ in the standard BT group. The change in AQLQ score 12 months post-BT was not significantly different between the MRI-guided and standard BT 


\begin{tabular}{|c|c|c|c|}
\hline & MRI-guided BT & Standard BT & p-value ${ }^{\#}$ \\
\hline Subjects $\mathrm{n}$ & 5 & 7 & \\
\hline Age years & $49 \pm 12$ & $42 \pm 14$ & 0.44 \\
\hline Female sex & $4(80)$ & $5(71)$ & ND \\
\hline $\mathrm{BMI} \mathrm{kg} \cdot \mathrm{m}^{-2}$ & $33 \pm 9$ & $29 \pm 5$ & 0.39 \\
\hline $\mathrm{BMI} \geqslant 30 \mathrm{~kg} \cdot \mathrm{m}^{-2}$ & $3(60)$ & $5(71)$ & ND \\
\hline Smoking history & $1(20)$ & $2(29)$ & ND \\
\hline Blood eosinophils $\times 10^{9}$ per $L$ & $0.3(0.0-0.6)$ & $0.2(0.0-0.7)$ & 0.86 \\
\hline Sputum eosinophils $\%$ & $0.7(0.0-1.5)^{\natural}$ & $0.2(0.0-4.3)^{+}$ & 0.90 \\
\hline ACQ-5 score & $2.8 \pm 1.1$ & $2.7 \pm 1.1$ & 0.88 \\
\hline AQLQ score & $3.4 \pm 1.6$ & $4.0 \pm 1.1$ & 0.48 \\
\hline $\mathrm{FEV}_{1}$ pre-BD \% pred & $44 \pm 8$ & $83 \pm 23$ & 0.005 \\
\hline $\mathrm{FEV}_{1}$ post-BD \% pred & $63 \pm 13$ & $92 \pm 22$ & 0.03 \\
\hline Reversibility of $\mathrm{FEV}_{1} \%$ & $51(6-87)$ & $11(-3-35)$ & 0.04 \\
\hline $\mathrm{FEV}_{1} / \mathrm{FVC}$ post-BD $\%$ & $63 \pm 14$ & $76 \pm 13$ & 0.12 \\
\hline MRI VDP pre-BD & $11 \pm 9$ & $13 \pm 17$ & 0.76 \\
\hline MRI VDP post-BD & $6 \pm 6$ & $8 \pm 11$ & 0.76 \\
\hline \multicolumn{4}{|l|}{ Asthma medications } \\
\hline ICS dose $\mu \mathrm{g} \cdot \mathrm{day}^{-1 \S}$ & $1500(1000-4000)$ & $1000(500-2000)$ & 0.27 \\
\hline OCS dose $\mathrm{mg} \cdot$ day $^{-1}$ & $0(0-20)$ & $0(0-25)$ & 0.84 \\
\hline OCS dependent & $1(20)$ & $2(29)$ & ND \\
\hline Monoclonal antibody & $3(60)$ & $1(14)$ & ND \\
\hline LABA & $5(100)$ & $7(100)$ & ND \\
\hline LAMA & $3(60)$ & $3(43)$ & ND \\
\hline LTRA & $3(60)$ & $4(57)$ & ND \\
\hline \multicolumn{4}{|c|}{$\begin{array}{l}\text { Data are presented as mean } \pm \text { SD, median (minimum-maximum) or } n(\%) \text {, unless otherwise indicated. Bold text } \\
\text { signifies a statistically significant difference. MRI: magnetic resonance imaging; BT: bronchial thermoplasty; ND: } \\
\text { not determined; BMI: body mass index; ACQ- } 5 \text { : five-item Asthma Control Questionnaire; AQLQ: Asthma Quality of } \\
\text { Life Questionnaire; FEV }_{1} \text { : forced expiratory volume in } 1 \mathrm{~S} \text {; BD: bronchodilator; FVC: forced vital capacity; VDP: } \\
\text { ventilation defect per cent; ICS: inhaled corticosteroid; OCS: oral corticosteroid; LABA: long-acting } \beta \text {-agonist; } \\
\text { LAMA: long-acting muscarinic antagonist; LTRA: leukotriene receptor antagonist. ": significance of difference } \\
\text { (p<0.05) between groups determined using an unpaired t-test for parametric data or Mann-Whitney test for } \\
\text { nonparametric data; }{ }^{\bullet}: \mathrm{n}=4 ;^{+}: \mathrm{n}=6 ;^{\S}: \text { fluticasone or equivalent. }\end{array}$} \\
\hline
\end{tabular}

groups $(\mathrm{p}=0.25$ ). In addition, the improvement in AQLQ score was $\geqslant$ MCID for four of five participants $(80 \%)$ in the MRI-guided group and five of seven participants $(71 \%)$ in the standard BT group at 12 months ( $\mathrm{p}>0.99)$.

Similar outcomes were observed for ACQ-5. As shown in figure 4b and supplementary figure S1B, the mean change in ACQ-5 score from baseline to 12 months post-BT was -1.4 (95\% CI $-2.6--0.2)(n=5$, $\mathrm{p}=0.03)$ in the MRI-guided BT group and $-0.7(95 \%$ CI $-1.3-0.0)(n=7, p=0.04)$ in the standard BT group. The change in ACQ-5 score 12 months post-BT was not significantly different between the MRI-guided and standard BT groups ( $\mathrm{p}=0.17$ ). The improvement in ACQ-5 score was $\geqslant$ MCID for four of five participants (80\%) in the MRI-guided group and four of seven participants (57\%) in the standard BT group at 12 months $(\mathrm{p}=0.58)$.

\section{Adverse events}

MRI and hyperpolarised gas inhalation were well tolerated by all participants. The total number of ${ }^{3} \mathrm{He}$ and ${ }^{129}$ Xe doses was 125 in 12 participants. There were 14 mild adverse events after dose inhalation in five of 12 participants (42\%), all of which resolved within 2 min of onset without treatment. Six adverse events in three participants were related to the breath-hold manoeuver (peripheral oxygen saturation $\left(S_{\mathrm{pO}_{2}}\right) \leqslant 85 \%$ for a few seconds after breath-hold) and eight adverse events in two participants (light headedness reported six times and tingling arms reported twice) were also judged related to the breath-hold manoeuver.

BT was well tolerated by all but one participant in the standard BT group, who required early termination of the first and third BT sessions owing to bronchospasm and wheeze. The most frequently observed adverse respiratory events are summarised in table 2 and hospitalisations for adverse respiratory events are detailed in supplementary table S2. During the intervention period (BT plus 6 weeks), there were two 


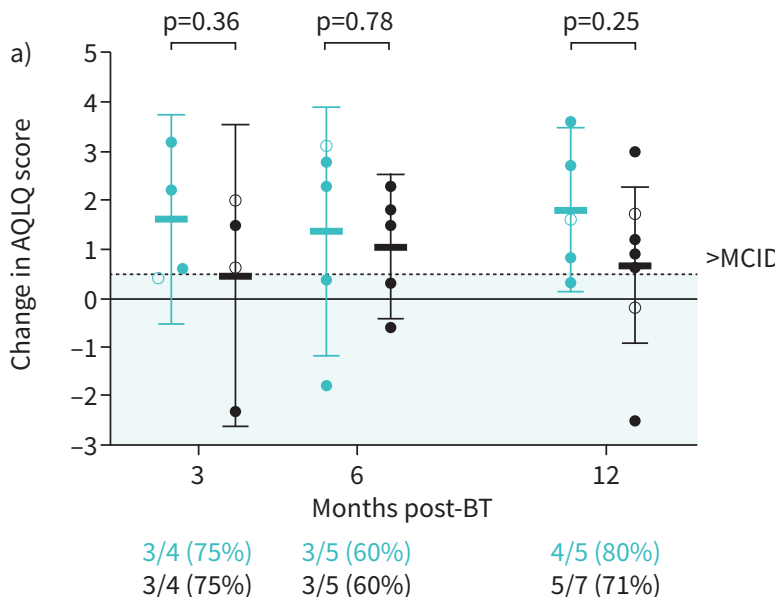

- MRI-guided BT

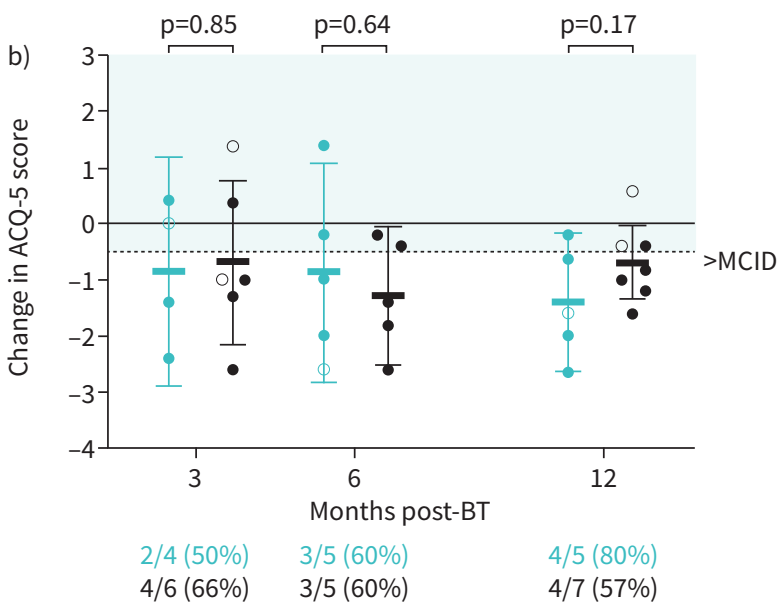

Standard BT

FIGURE 4 Change in Asthma Quality of Life Questionnaire (AQLQ) score (a) and five-item Asthma Control Questionnaire (ACQ-5) score (b) following magnetic resonance imaging (MRI)-guided (teal) and standard (black) bronchial thermoplasty (BT). Bars show the mean change from baseline and 95\% confidence intervals with individual values for all participants superimposed on the plot. Open and solid symbols represent participants who underwent BT at site 1 and site 2, respectively. The number and proportion of participants with an AQLQ score and ACQ-5 score improvement $\geqslant$ minimal clinically important difference are provided below each plot.

hospitalisations for adverse respiratory events in each group. In the MRI-guided BT group, two of five participants (40\%) required a total of two hospitalisations (one 7 days after BT for 12 days; one within 1 day after BT for 3 days). In the standard BT group, one of seven participants (14\%) required two hospitalisations (both within 1 day after BT, for 5 and 21 days). A similar proportion of participants in both groups were hospitalised for adverse respiratory events during the post-intervention period, although hospitalisations were less frequent in the MRI-guided BT group (one participant (20\%) required one hospitalisation) than in the standard group (two participants (29\%) required a total of four hospitalisations). The total duration of hospitalisation was 20 days in the MRI-guided BT group and 41 days in the standard BT group.

\section{Discussion}

For current and newly emerging asthma therapies, the assumption has been that airway abnormalities are homogenously distributed. Recent work has shown that asthma airway abnormalities are, in fact, regionally heterogeneous, and this observation can be exploited to guide therapies to only the abnormal airways. Because BT targets all accessible airways with curative intent, guided therapies may offer potential advantages over standard BT.

In this pilot randomised controlled trial, MRI-guided BT resulted in clinically and statistically significant improvements in asthma-specific quality of life and control over 12 months. Despite receiving 53\% fewer radiofrequency activations, four of five participants (80\%) who underwent MRI-guided BT reported a clinically relevant AQLQ and ACQ-5 score improvement at 12 months. This response rate is similar to the AIR-2 trial (79\%) [8] and the Australian Bronchial Thermoplasty Registry (84\%) [12]. At 12 months, we reported a mean AQLQ and ACQ-5 score improvement of 1.8 and -1.4, respectively, which is statistically significant and consistent with published trials [6-8]. Importantly, we made a number of observations that suggest these improvements were non-inferior to standard BT. First, the improvement from baseline in AQLQ and ACQ-5 scores were not statistically different between the intervention groups. Second, the proportion of AQLQ and ACQ-5 responders was similar between intervention groups. Finally, despite our small sample size, the upper bound of the 95\% CI (-1.8-0.4) around the difference between intervention groups with respect to ACQ-5 score improvement at 12 months was below the non-inferiority margin of 0.5 points.

Our observations support and complement the previous findings of HALL et al. [28], which also showed that $>70 \%$ of MRI-guided BT participants reported a clinically relevant AQLQ score improvement at 3 months. While this previous work [28] was not designed to evaluate the effectiveness of MRI-guided BT beyond 3 months, our trial confirmed that such improvements were durable 12 months post-BT. In both 


\section{TABLE 2 Proportion of participants with adverse respiratory events}

MRI-guided BT

\begin{tabular}{|lll|}
\hline Intervention period plus 6 weeks & & \\
\hline SAE - hospitalisation & $2 / 5(40 \%)$ & $1 / 7(14 \%)$ \\
\hline Cough & $4 / 5(80 \%)$ & $6 / 7(86 \%)$ \\
\hline Wheeze & $3 / 5(60 \%)$ & $4 / 7(57 \%)$ \\
\hline Chest tightness & $1 / 5(20 \%)$ & $4 / 7(57 \%)$ \\
\hline Consolidation/atelectasis/airspace opacity & $2 / 5(40 \%)$ & $4 / 7(57 \%)$ \\
\hline Dyspnoea & $2 / 5(40 \%)$ & $3 / 7(43 \%)$ \\
\hline Chest discomfort & $1 / 5(20 \%)$ & $3 / 7(43 \%)$ \\
\hline Bronchospasm & $1 / 5(20 \%)$ & $3 / 7(43 \%)$ \\
\hline Haemoptysis & $0 / 5(0 \%)$ & $3 / 7(43 \%)$ \\
\hline Productive cough & $1 / 5(20 \%)$ & $3 / 7(43 \%)$ \\
\hline Decreased breath sounds & $2 / 5(40 \%)$ & $2 / 7(29 \%)$ \\
\hline Sputum discoloured & $2 / 5(40 \%)$ & $0 / 7(0 \%)$ \\
\hline Infection & $2 / 5(40 \%)$ & $1 / 7(14 \%)$ \\
\hline Fever & $1 / 5(20 \%)$ & $1 / 7(14 \%)$ \\
\hline Night awakenings & $0 / 5(0 \%)$ & $1 / 7(14 \%)$ \\
\hline Bronchospasm & $0 / 5(0 \%)$ & $1 / 7(14 \%)$ \\
\hline Post-intervention period (6 weeks-12 months) & & \\
\hline SAE - hospitalisation & $1 / 5(20 \%)$ & $2 / 7(29 \%)$ \\
\hline Cough & $2 / 5(40 \%)$ & $4 / 7(57 \%)$ \\
\hline Dyspnoea & $3 / 5(60 \%)$ & $4 / 7(57 \%)$ \\
\hline Chest tightness & $1 / 5(20 \%)$ & $4 / 7(57 \%)$ \\
\hline Wheeze & $2 / 5(40 \%)$ & $3 / 7(43 \%)$ \\
\hline Productive cough & $1 / 5(20 \%)$ & $3 / 7(43 \%)$ \\
\hline Infection & $1 / 5(20 \%)$ & $3 / 7(43 \%)$ \\
\hline Chest discomfort & $0 / 5(0 \%)$ & $2 / 7(29 \%)$ \\
\hline Night awakenings & $0 / 5(0 \%)$ & $2 / 7(29 \%)$ \\
\hline Sputum discoloured & $1 / 5(20 \%)$ & $1 / 7(14 \%)$ \\
\hline Decreased breath sounds & $1 / 5(20 \%)$ & $1 / 7(14 \%)$ \\
\hline Fever & $0 / 5(0 \%)$ & $1 / 7(14 \%)$ \\
\hline Data are presented as n/N (\%). MRI: magnetic resonance & & \\
adverse event. & BT: bronchial thermoplasty; SAE: severe \\
\hline & & \\
\hline
\end{tabular}

trials, outcomes were not related to the number of radiofrequency activations, contradicting the observations by LANGTON et al. [29] that fewer activations were associated with poor response following standard BT. It is important to highlight a number of methodological differences between the trials. First, different criteria were used to identify treatment targets on MRI. Hall et al. [28] employed an MRI-CT co-registration and segmentation approach to quantify segmental VDP from up to four baseline ${ }^{129}$ Xe MRI scans and targeted the six bronchopulmonary segments with the largest mean VDP in a single bronchoscopy session. By contrast, we visually inspected the dynamic behaviour of ventilation defects in response to bronchodilator to identify targets. We did not impose an upper limit on the number of targets, which led to the treatment of four to seven segments during a single procedure and some participants requiring two bronchoscopy sessions to complete MRI-guided BT. It is logical to assume that the aforementioned discrepancies in terms of the number and characteristics of treatment targets, as well as in the approach to localise targets (automated imaging processing versus visual interpretation by bronchoscopist), may influence the efficacy of MRI-guided BT and highlight the need for mechanistic studies to optimise treatment decisions. An advanced CT-MRI registration and segmentation approach, similar to that employed by HaLl et al. [28], would likely be a more repeatable and scalable means to localise treatment targets.

We must acknowledge a number of assumptions when identifying patient-specific airway targets. In the MRI-guided BT group, we elected to guide BT to airways that were spatially aligned with ventilation defects that improved following bronchodilator, which we believe reflected ASM dysfunction [19]. We prospectively planned to avoid treating airways that were spatially aligned to "persistent" or "bronchodilator non-responsive" ventilation defects because we have previously shown that these are the consequence of intraluminal plugging due to inflammatory cells [19, 36] and/or mucus [18]. Importantly, there was a single protocol deviation in the MRI-guided BT group (subject-11) for a participant with 
persistent lower lobe ventilation defects in whom the interventional specialist treated all 10 lower lobe segments. Retrospective review of the chest CT revealed mucus plugs in two (RB6 and LB6) of the 10 bronchopulmonary segments that were treated. Our approach assumed 1) adequate inhaled bronchodilator deposition to all segmental airways and 2) MRI ventilation defects that stemmed from intraluminal plugging or other mechanisms not responsive to bronchodilator were not good targets for BT. While the optimal treatment target has not yet been established, our decision-making was informed by the primary mechanism of action of BT (reduced ASM mass [2-5]) and our understanding of the underlying contributions to MRI ventilation defects. Nevertheless, it is important to emphasise that bronchial biopsy [3] and modelling studies [37] suggest that BT effects are not localised to the ASM or the site of radiofrequency delivery and may have effects on other structural cells such as the epithelium [38] or nerves [5, 39]. Pretolani et al. [3] observed reduced ASM area in the untreated right middle lobe of participants who underwent BT, suggesting that thermal injury may cross lobar divisions and affect adjacent lobes. In addition, while BT directly treats only the large airways, Donovan et al. [37] provided evidence via a mathematical model that structural changes in the large airways leads to reopening of the smaller airways via a global flow redistribution mechanism. SALEM et al. [38] observed decreased mucin 5AC and interleukin-13 expression 1 year after BT, which challenges our assumption that ventilation defects distal to mucus plugs are not ideal targets for BT. It is unclear if BT consistently normalises interleukin-13 activity and mucus in all patients; this requires further investigation.

There are a number of limitations to our pilot trial that should be considered. We acknowledge that the small sample size likely contributed to different clinical characteristics between intervention groups at baseline. At baseline, the MRI-guided BT group were treated with more biologics, had lower FEV 1 and greater bronchodilator reversibility. While AQLQ and ACQ-5 scores were similar between the groups, we did not account for baseline differences in severity or lung function between the groups and cannot exclude that the standard BT group would have shown better outcomes if it had consisted of patients with "equally severe" asthma. We estimate that only 5-8\% of all patients with severe asthma are optimal candidates for BT; therefore, our small sample size is a consequence of the small number of patients clinically selected to receive BT at the study sites. During the study period, only two patients underwent BT outside of the study protocol. Moreover, our study was not blinded because we did not implement a sham procedure in the MRI-guided BT group to mimic standard BT of all airways. Therefore, both the participant and clinical team were aware of intervention allocation during the post-intervention period. This may be problematic given the subjective nature of our end-points. Finally, treatment targets for all but one participant were identified on MRI using hyperpolarised ${ }^{3} \mathrm{He}$ gas, not ${ }^{129} \mathrm{Xe}$ gas. Because the hyperpolarised gas community has now transitioned to the use of ${ }^{129} \mathrm{Xe}$ gas to reduce costs and improve accessibility to hyperpolarised gas MRI, documented biases between the gases [23, 40, 41] should be considered when generalising our trial findings.

In summary, we completed a 1-year pilot randomised controlled trial and observed that MRI-guided BT resulted in a reduced number of BT activations and bronchoscopy sessions, with clinically and statistically significant improvements in asthma control and quality of life at 1 year that were non-inferior to standard whole-lung BT. A patient-specific approach to BT offers advantages over standard BT in terms of reduced procedure time, cost and adverse events, and perhaps enables BT in patients with more severe asthma.

Acknowledgements: We thank Dante Capaldi and Heather Young for production and dispensing of hyperpolarised gas, Kiran Kooner for quantitative MRI analysis and David Reese for MRI of research volunteers (Robarts Research Institute, Western University, London, Canada).

Provenance: Submitted article, peer reviewed.

This study is registered at www.clinicaltrials.gov with identifier number NCT02263794.

Data availability: Because this clinical trial began enrolling participants prior to January 1 , 2019, a data sharing plan was not put in place in the trial's registration.

Conflict of interest: S. Svenningsen reports personal fees from AstraZeneca and Novartis, grants from Cyclomedica and personal fees from Polarean, outside the submitted work. P. Nair reports grants and personal fees from AstraZeneca, grants from Novartis, grants and personal fees from Teva, grants from Sanofi, grants and personal fees from Roche, personal fees from Novartis, Merck and Equillium, and grants from Foresee, outside the submitted work. R.L Eddy has nothing to disclose. M.J. McIntosh has nothing to disclose. M. Kjarsgaard has 
nothing to disclose. H.F. Lim has nothing to disclose. D.G. McCormack has nothing to disclose. G. Cox has nothing to disclose. G. Parraga reports grants from Astra Zeneca and Novartis, outside the submitted work.

Support statement: S. Svenningsen was supported by Canadian Institutes of Health Research (CIHR) Banting and Canadian Respiratory Research Network (CRRN) postdoctoral fellowship awards. P. Nair was supported by the Frederick E. Hargreave Teva Innovation Chair in Airway Diseases. R.L. Eddy was supported by a National Science and Engineering Research Council postgraduate doctoral award. The study was supported by CIHR and CRRN operating grant funding and grants from AllerGen NCE. G. Parraga was supported by the Canada Research Chair Program. Funding information for this article has been deposited with the Crossref Funder Registry.

\section{References}

1 Cox PG, Miller J, Mitzner W, et al. Radiofrequency ablation of airway smooth muscle for sustained treatment of asthma: preliminary investigations. Eur Respir J 2004; 24: 659-663.

2 Goorsenberg AWM, d'Hooghe JNS, Srikanthan K, et al. Bronchial thermoplasty induced airway smooth muscle reduction and clinical response in severe asthma. The TASMA randomized trial. Am J Respir Crit Care Med 2021; 203: 175-184.

3 Pretolani M, Dombret MC, Thabut G, et al. Reduction of airway smooth muscle mass by bronchial thermoplasty in patients with severe asthma. Am J Respir Crit Care Med 2014; 190: 1452-1454.

4 Salem IH, Boulet LP, Biardel S, et al. Long-term effects of bronchial thermoplasty on airway smooth muscle and reticular basement membrane thickness in severe asthma. Ann Am Thorac Soc 2016; 13: 1426-1428.

5 Jendzjowsky $\mathrm{N}$, Laing $\mathrm{A}$, Malig $\mathrm{M}$, et al. Long-term modulation of airway remodeling in severe asthma following bronchial thermoplasty. Eur Respir J 2021; 58: 2100622.

6 Cox G, Thomson NC, Rubin AS, et al. Asthma control during the year after bronchial thermoplasty. N Engl J Med 2007; 356: 1327-1337.

7 Pavord ID, Cox G, Thomson NC, et al. Safety and efficacy of bronchial thermoplasty in symptomatic, severe asthma. Am J Respir Crit Care Med 2007; 176: 1185-1191.

8 Castro M, Rubin AS, Laviolette M, et al. Effectiveness and safety of bronchial thermoplasty in the treatment of severe asthma: a multicenter, randomized, double-blind, sham-controlled clinical trial. Am J Respir Crit Care Med 2010; 181: 116-124.

9 Wechsler ME, Laviolette M, Rubin AS, et al. Bronchial thermoplasty: long-term safety and effectiveness in patients with severe persistent asthma. J Allergy Clin Immunol 2013; 132: 1295-1302.

10 Castro M, Rubin A, Laviolette M, et al. Persistence of effectiveness of bronchial thermoplasty in patients with severe asthma. Ann Allergy Asthma Immunol 2011; 107: 65-70.

11 Chupp G, Laviolette M, Cohn L, et al. Long-term outcomes of bronchial thermoplasty in subjects with severe asthma: a comparison of 3-year follow-up results from two prospective multicentre studies. Eur Respir J 2017; 50: 1700017

12 Langton D, Wang W, Sha J, et al. Predicting the response to bronchial thermoplasty. J Allergy Clin Immunol Pract 2020; 8: 1253-1260.

13 Chaudhuri R, Rubin A, Sumino K, et al. Safety and effectiveness of bronchial thermoplasty after 10 years in patients with persistent asthma (BT10+): a follow-up of three randomised controlled trials. Lancet Respir Med 2021; 9: 457-466.

14 Nguyen HV, Bose S, Mital S, et al. Is bronchial thermoplasty cost-effective as treatment for problematic asthma patients? Singapore's perspective on a global model. Respirology 2017; 22: 1102-1109.

15 Zafari Z, Sadatsafavi M, Marra CA, et al. Cost-effectiveness of bronchial thermoplasty, omalizumab, and standard therapy for moderate-to-severe allergic asthma. PLoS One 2016; 11: e0146003.

16 Eddy RL, Svenningsen S, Kassay A, et al. This is what asthma looks like: review of new and emerging functional imaging methods and results. Can J Respir Crit Care Sleep Med 2018; 2: 27-40.

17 Svenningsen S, Kirby M, Starr D, et al. What are ventilation defects in asthma? Thorax 2014; 69: 63-71.

18 Svenningsen S, Haider E, Boylan C, et al. CT and functional MRI to evaluate airway mucus in severe asthma. Chest 2019; 155: 1178-1189.

19 Svenningsen S, Eddy RL, Lim HF, et al. Sputum eosinophilia and magnetic resonance imaging ventilation heterogeneity in severe asthma. Am J Respir Crit Care Med 2018; 197: 876-884.

20 Costella S, Kirby M, Maksym GN, et al. Regional pulmonary response to a methacholine challenge using hyperpolarized ${ }^{3} \mathrm{He}$ magnetic resonance imaging. Respirology 2012; 17: 1237-1246.

21 Kruger SJ, Niles DJ, Dardzinski B, et al. Hyperpolarized helium-3 MRI of exercise-induced bronchoconstriction during challenge and therapy. J Magn Reson Imaging 2014; 39: 1230-1237.

22 Samee $\mathrm{S}$, Altes T, Powers $\mathrm{P}$, et al. Imaging the lungs in asthmatic patients by using hyperpolarized helium-3 magnetic resonance: assessment of response to methacholine and exercise challenge. J Allergy Clin Immunol 2003; 111: 1205-1211.

23 Svenningsen S, Kirby M, Starr D, et al. Hyperpolarized ${ }^{3} \mathrm{He}$ and ${ }^{129} \mathrm{Xe}$ MRI: differences in asthma before bronchodilation. J Magn Reson Imaging 2013; 38: 1521-1530. 
de Lange EE, Altes TA, Patrie JT, et al. Evaluation of asthma with hyperpolarized helium-3 MRI: correlation with clinical severity and spirometry. Chest 2006; 130: 1055-1062.

Mummy DG, Carey KJ, Evans MD, et al. Ventilation defects on hyperpolarized helium-3 MRI in asthma are predictive of 2-year exacerbation frequency. J Allergy Clin Immunol 2020; 146: 831-839.

Svenningsen S, Nair P, Guo F, et al. Is ventilation heterogeneity related to asthma control? Eur Respir J 2016; 48: 370-379.

Thomen RP, Sheshadri A, Quirk JD, et al. Regional ventilation changes in severe asthma after bronchial thermoplasty with ${ }^{3} \mathrm{He}$ MR imaging and CT. Radiology 2015; 274: 250-259.

Hall CS, Quirk JD, Goss CW, et al. Single-session bronchial thermoplasty guided by ${ }^{129}$ Xe magnetic resonance imaging. A pilot randomized controlled clinical trial. Am J Respir Crit Care Med 2020; 202: 524-534.

Langton D, Sha J, Ing A, et al. Bronchial thermoplasty: activations predict response. Respir Res 2017; 18: 134.

Chung KF, Wenzel SE, Brozek JL, et al. International ERS/ATS guidelines on definition, evaluation and treatment of severe asthma. Eur Respir J 2014; 43: 343-373.

Kirby M, Heydarian M, Svenningsen S, et al. Hyperpolarized ${ }^{3} \mathrm{He}$ magnetic resonance functional imaging semiautomated segmentation. Acad Radiol 2012; 19: 141-152.

Cox G, Miller JD, McWilliams A, et al. Bronchial thermoplasty for asthma. Am J Respir Crit Care Med 2006; 173: 965-969.

Juniper EF, Svensson K, Mork AC, et al. Measurement properties and interpretation of three shortened versions of the asthma control questionnaire. Respir Med 2005; 99: 553-558.

Juniper EF, Guyatt GH, Willan A, et al. Determining a minimal important change in a disease-specific quality of life questionnaire. J Clin Epidemiol 1994; 47: 81-87.

Svenningsen S, Lim HF, Goodwin S, et al. Optimizing sputum cell counts prior to bronchial thermoplasty: a preliminary report. Can J Respir Crit Care Sleep Med 2019; 3: 143-147.

Svenningsen S, Eddy RL, Kjarsgaard M, et al. Effects of anti-T2 biologic treatment on lung ventilation evaluated by MRI in adults with prednisone-dependent asthma. Chest 2020; 158: 1350-1360.

Donovan GM, Elliot JG, Green FHY, et al. Unraveling a clinical paradox: why does bronchial thermoplasty work in asthma? Am J Respir Cell Mol Biol 2018; 59: 355-362.

Haj Salem I, Gras D, Joubert P, et al. Persistent reduction of mucin production after bronchial thermoplasty in severe asthma. Am J Respir Crit Care Med 2019; 199: 536-538.

9 Ichikawa T, Panariti A, Audusseau S, et al. Effect of bronchial thermoplasty on structural changes and inflammatory mediators in the airways of subjects with severe asthma. Respir Med 2019; 150: 165-172.

40 Stewart NJ, Chan HF, Hughes PJC, et al. Comparison of ${ }^{3} \mathrm{He}$ and ${ }^{129} \mathrm{Xe}$ MRI for evaluation of lung microstructure and ventilation at 1.5 T. J Magn Reson Imaging 2018; 48: 632-642.

Kirby M, Svenningsen S, Owrangi A, et al. Hyperpolarized ${ }^{3} \mathrm{He}$ and ${ }^{129} \mathrm{Xe}$ MR imaging in healthy volunteers and patients with chronic obstructive pulmonary disease. Radiology 2012; 265: 600-610. 